\title{
Voltage Collapse in Power Systems
}

\author{
Circuit and system techniques for analyzing \\ voltage collapse are moving toward \\ practicalapplication- and none too soon

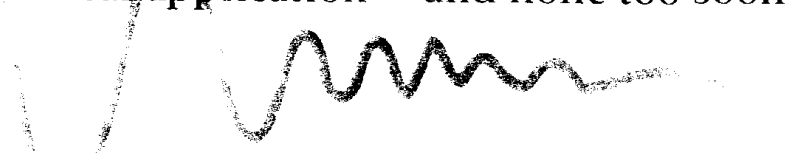

by Ian Dobson, Hans Glavitsch, Chen-Ching Liu, Yasuo Tamura, and Khoi Vu

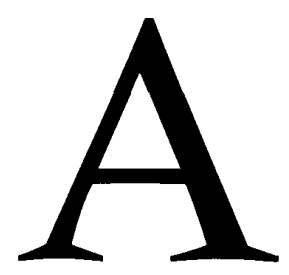

s power systems become more complex and more heavily loaded, voltage collapse becomes an increasingly serious problem. Voltage collapse- with its associate power blackout - has already occurred in real-world electric power systems. Fortunately, practical analytical tools will soon be making their ways from researchers to system designers and operators.

A power system is an electrical network containing components such as generators, transmission lines, loads, and voltage controllers (Fig. 1). Practical networks are large, ranging from hundreds to thousands of nodes and branches. Since the basic practical functions of a power system are the production and distribution of electrical power, generators are essential components. Under normal operating conditions, a generator is essentially a constant-voltage source. But in a transient condition, the excitation and rotor dynamics can produce undesirable oscillations of the system frequency and voltage magnitude.

As for the components of the system, a transmission line can be modeled by a series $R L$ branch with shunt capacitors. The control system is critical, and has benefitted from recent technological advances. The turns-ratios of some transformers, for example, are automatically adjusted by onload tap-changers (OLTCs) to maintain the voltage levels near the load. But although control mechanisms have increased in number and sophistication, the networks themselves are very complex and can behave in ways that are difficult to predict.

Load devices themselves vary greatly, from resistive lighting devices to dynamic components such as large induction motors. And power systems can be interconnected to allow exchange of electrical power between different utility systems. A large, nonlinear, interconnected power network can exhibit very complex dynamic phenomena when the system is disturbed from a steady-state operating condition.
To complicate things even more, power systems are becoming more heavily loaded as the demand for electric power rises, while economic and environmental concerns limit the construction of new transmission and generation capacity. Under these stressful operating conditions, we are encountering a new instability problem called voltage collapse, which has led to blackouts in electric utilities around the world $[1,2,3]$.

\section{How Voltages Collapse}

In the initial stage of a voltage collapse, voltage magnitudes at substations fall gradually. There may be temporary voltage recovery due to control actions, but the voltages soon fall to very low levels nonetheless (Fig. 2). Protective devices in the power system may observe the abnormal condi-

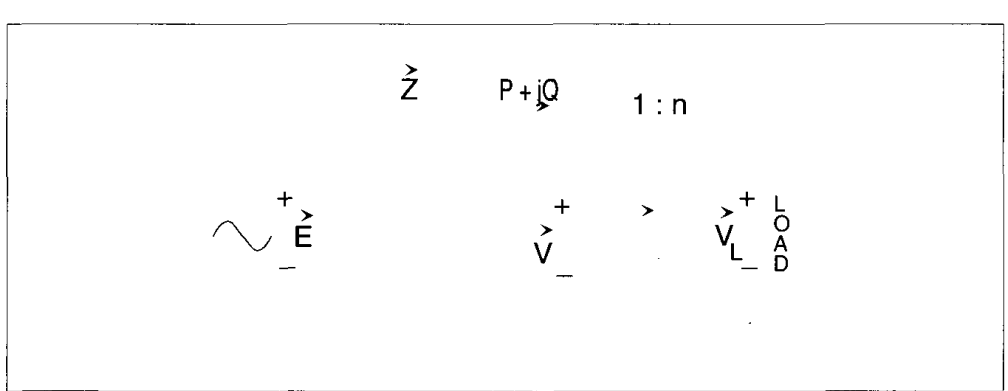

1. A simple power system with all of its essential components: generator, transmission line, voltage controller (OLTC), and load. 


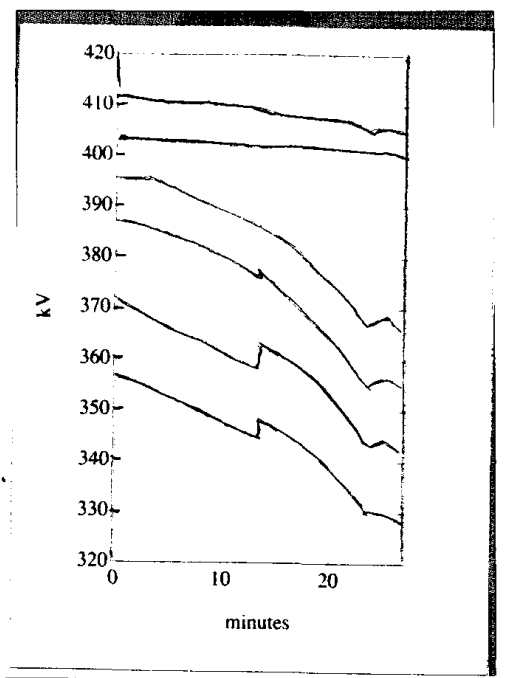

2. An example of voltage collapse in a $400-\mathrm{kV}$ power system [1]. modeling of the generator's voltage control and load dynamics may be simplified or ignored, even in numerical simulations. These are precisely the considerations that must be incorporated in a realistic yet tractable model for analytical studies of voltage collapse.

The second issue is the development of voltage-collapse analytical methods. We now understood that voltage collapse is a dynamic problem, some aspects of which can be captured by steady-state models. A network's power-flow equations, for example, are derived from the steady-state $\mathrm{Kir}$ chhoff's laws for the network. In simple terms, voltage collapse is caused by the loss of such a steady-state solution. Voltage collapse can thus be related to the changing number of solutions for the power-flow equations, due to slow changes in system parameters. To fully analyze voltage col- introduce the essential ideas. Consider a nonlinear system with a single state variable, $x$, and a slowly varying parameter, $\lambda$. The dynamics when $\lambda=-2$ are shown by the corresponding vertical line in Fig. 3.

There are two equilibria, i.e., steady state solutions, labeled $x_{s}$ and $x_{u}$. The dynamics at other points are indicated by the arrows. The variable $x_{s}$ is a stable equilibrium, since the dynamics tend to return $x$ to $x_{s}$ if the system state $x$ is perturbed away from $x_{s}$. The variable $x_{u}$, on the other hand, is an unstable equilibrium. In normal system operation, the system state $x$ is at or near the stable equilibrium.

As $\lambda$ varies slowly, the positions of the equilibria $x_{s}$ and $x_{u}$ vary slowly (see the different positions of the equilibria for $\lambda=$ -1 ), and the system state will "track" $x_{s}$ because $x_{s}$ is stable. Thus, for normal operation, it is reasonable to assume that the system state is always at the stable equilibrium $x_{s}$, and that a steady-state model can therefore be ușed for analysis.

For negative values of $\lambda$, the system always maintains one stable and one unstable equilibrium point, but if $\lambda$ reaches zero, $x_{s}$ and $x_{u}$ meet to form a single equilibrium, $x *$. There is no equilibrium for positive values of $\lambda$. This qualitative change in the number of equilibria is called bifurcation. The critical $\lambda$ values at which bifurcation occurs are called the bifurcation set. In this system the bifurcation set is the single point, $\lambda=0$.

The steady-state model of $x$ tracking $x_{s}$ is not applicable when $\lambda$ reaches its critical value because $x *$ is unstable. The slightest perturbation decreasing the state will cause $x$ to decrease dynamically. At $\lambda=1$, the $\mathrm{x}$ value drops monotonically and the loss of stability leads to a dynamic collapse. In summary, while $\lambda$ is negative, the system can be operated at the stable equilibrium $x_{s}$, but when $\lambda$ becomes zero the system becomes unstable and it "collapses."

Few engineering applications of bifurcations are one-dimensional but, fortunately, higher-dimensional bifurcations contain the one-dimensional case. For the power system of Fig. 1, the system state vector $x=V=(V, \delta)$ specifies the voltage phasor magnitude $V$, and angle $\delta$. (In this example, the parameter $\lambda$ varies slowly enough so that the secondary-side voltage $V_{L}$ always stays at a constant level thanks to the tap-changing transformer in Fig. 1. In such a case, only the primary-side voltage $V$ is of interest. When a large disturbance, i.e., a sudden change in $\lambda$ occurs, it takes time for the transformer to
Traditional power-system stability analysis is primarily concerned with rotorand frequency-oscillation problem. Hence, lapse for small- and large-disturbance cases mechanisms.

\section{Losing equilibrium}

Large-scale electric power systems are typinemear systems that are vary, such a system can un a qualitative change in which the system's stable operating point disappears. One consequence of an electric power system losing equilibrium is a decrease in system voltage leading to a voltage collapse [1].

Let's look at a prototypical example to 
restore the voltage $V_{L}$. The evolution of the system during this dynamical process is dis cussed in the section on dynamic mechanisms.)

The system parameter $\lambda$ can be chosen to measure the amount of system loading so that the load real and reactive powers are $\lambda P$ and $\lambda Q$. The static variation of the equilibria in this case is a curve in the three dimensions $V, \delta$, and $\lambda$ (Fig. 4). One curve shows the $V$ component of the equilibria as a function of $\lambda$; the other curve shows the $\delta$ component as a function of $\lambda$. The dynamics of the system are such that the upper branch of the $V$ curve corresponds to stable equilibria $x_{S}$, and the lower branch corresponds to unstable equilibria $x_{u}$. (Since $\delta$ increases as $V$ decreases, the upper branch of the $\delta$ curve corresponds to unstable equilibria and the lower branch corresponds to stable equilibria.) The bifur-

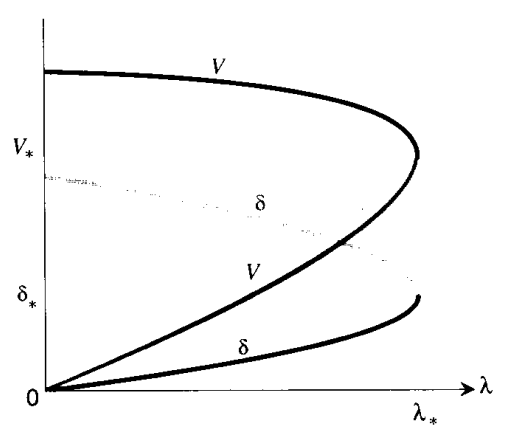

4. Bifurcation diagram for the example power system. $V$ and $\delta$ are the magnitude, and phase-shift angle, respectively, of the load voltage $\boldsymbol{V}$. before bifurcation

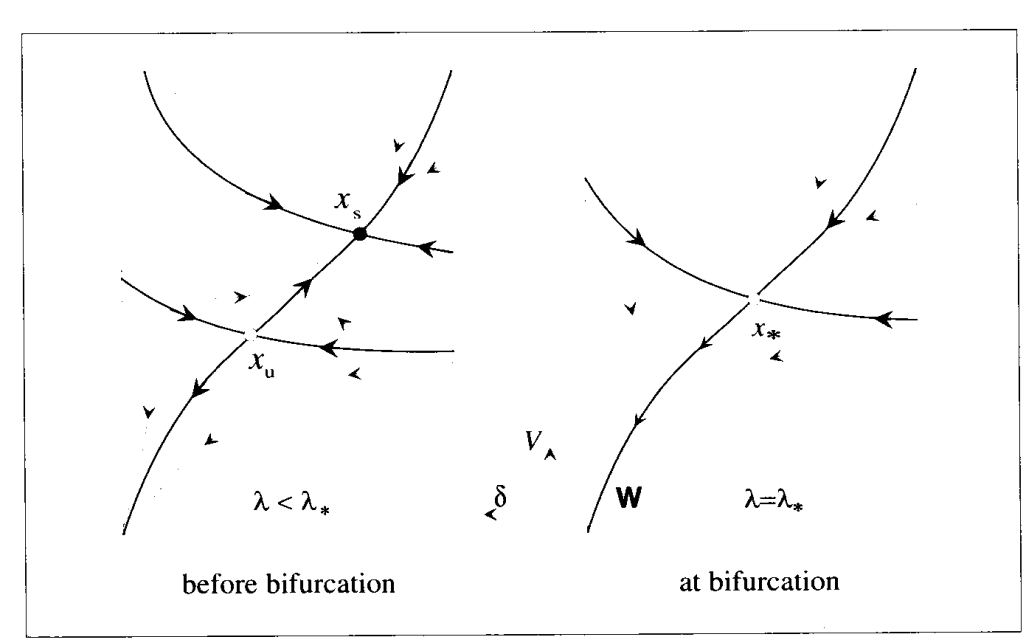

at bifurcation

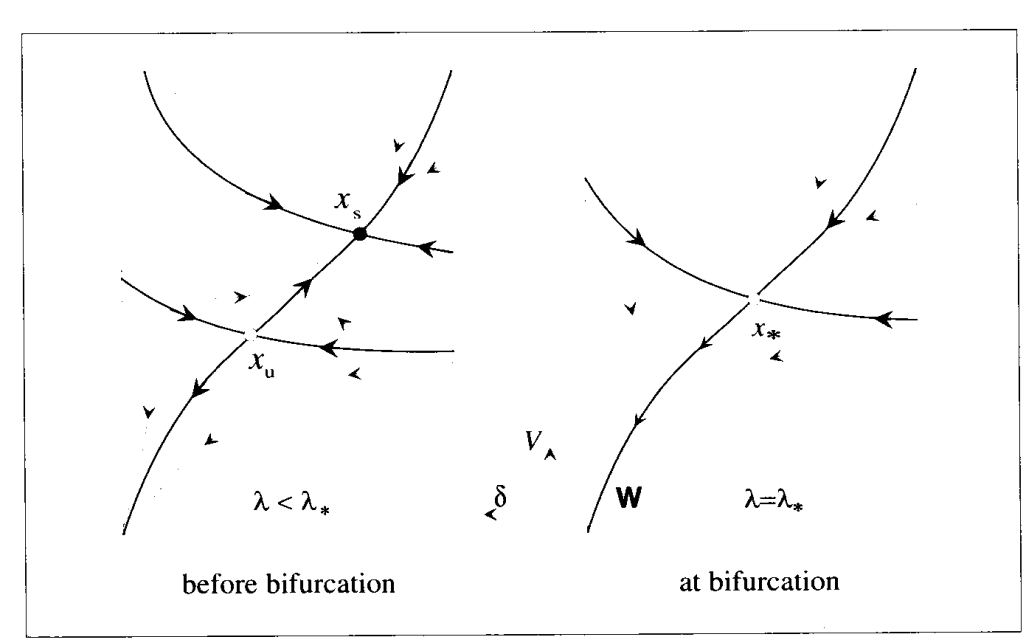

\section{The focus is on two issues, of which the first is modeling}

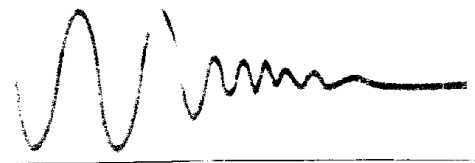

cation occurs at the loading $\lambda=\lambda_{*}$, where the equilibria $x_{s}$ and $x_{u}$ coalesce to form the unstable equilibrium $x_{*}=\left(V_{*}, \delta_{*}\right)$.

The loss of stability can also be viewed in the state space $(V, \delta)$ (Fig. 5). The dynamics are indicated by the arrows. Before
5. State space dynamics before and at bifurcation the bifurcation, $\lambda<\lambda *$ and the system is operated at the stable equilibrium $x_{s}$. The nearby equilibrium $x_{u}$ is unstable. (The system trajectories around $x_{s}$ converge to $x_{s}$, but those in the neighborhood of $x_{u}$ generally diverge from $x_{u}$.)

When $\lambda$ increases to $\lambda_{*}, x_{s}$ and $x_{u}$ coalesce to form $x *$. Since the system state $x$ was tracking $x_{5}, x$ finds itself initially at $x *$. An examination of the dynamics near $x *$ shows that $x *$ is unstable along the trajectory direction labeled $W$. The system dynamics make trajectories tend to this direction rapidly, and the movement in this direction explains why voltages can dynamically decline during voltage collapse.

The example of Fig. 5 shows a prototypical bifurcation with a single varying parameter for a system with two states $(V, \delta)$. The next step is to choose two parameters for the loading of the example power system to better illustrate the effect of multiple, independently varying parameters. This may be done by setting $\lambda$ as the vector of load powers $(P, Q)$. A given load pattern is then represented by a point $\lambda=(P, Q)$ (Fig. $6)$.

For normal operation, $\lambda$ lies in the shaded area, and there is a stable equilibrium $x_{s}$ in state space corresponding to $\lambda$ at which the system is operated. The curve in Fig. 6 is called the bifurcation set, and it is the set of critical loadings $\lambda_{*}=\left(P *, Q^{*}\right)$ at which the system has a bifurcation. There is no equilibrium for load patterns outside the bifurcation set.

Generalization from a system with two states and two loading parameters to an arbitrary power system with many states and loading parameters is conceptually straightforward, so the simple qualitative picture of voltage collapse outlined above is preserved in settings with more dimensions. The analytic and computational difficulties, however, are greatly increased and still challenge the ingenuity of people in the field.

The aforementioned theory of voltage collapse applies to any power system modeled by differential equations with slowly moving parameters, and it can be justified with generic bifurcation theory. A power system may be operated to avoid bifurcation and voltage collapse by monitoring the distance of the present loading $\lambda$ to the bifurcation set, and using available control mechanisms to increase the distance if it becomes too small. Controls may be thought of as modifying the bifurcation set. Much current research concerns defining and com- 


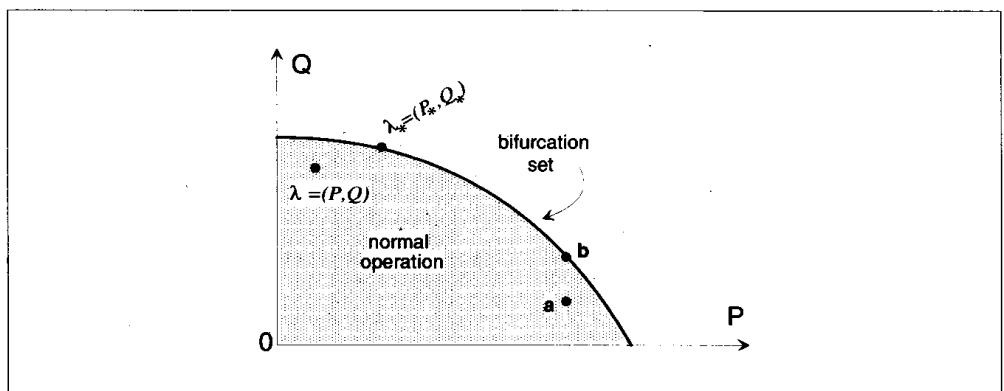

6. Two-dimensional load power parameter space. The parameter is $\lambda=(P, Q)$.

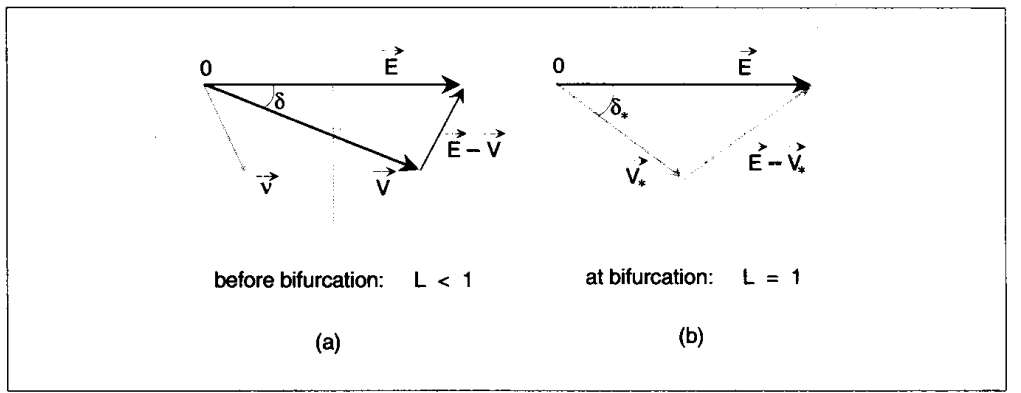

7. Voltage phasors before and at bifurcation. Comparing the sending and receiving voltages of the power system provides a measure of how close the operating equilibrium is from the point of bifurcation. The variables are defined by Fig. 1 .

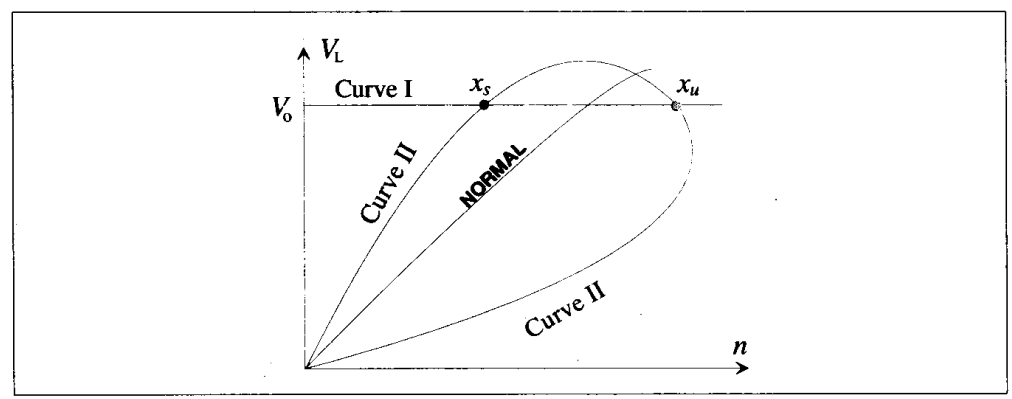

8. Partition of state space based on each mechanism.

puting various measures of the "distance" to bifurcation and developing appropriate control actions.

\section{Detecting Voltage Collapse}

Several algorithms have been developed to detect how close a system is to voltage collapse. All of these algorithms assess the distance between the present loading and the bifurcation set in the space of load demands. Figure 6 is a two-dimensional example.

Two typical indices of this distance are the $L$ index and the voltage instability operation $x_{u}$ by $v$. The index $L$ is defined as

$$
L=\frac{\text { voltage drop }}{\text { voltage received }}=\frac{|E-\eta|}{|\eta|}
$$

or $L=11-\mathrm{E} / \mathrm{Vl}$. Geometry suggests that $L<$ 1 for the stable voltage solution $V$. Bifurcation occurs when $V$ and $v$ coincide (Fig. 7b). $L=1$ at the bifurcation, and the closer $L$ is to 1 , the closer the system is to voltage collapse.

The concept underlying the VIPI can also be explained by reference to Fig. 7a. The dashed line that bisects $\boldsymbol{E}$ corresponds to the bifurcation set of Fig. 6. The average $(V+v) / 2$ is a voltage phasor having its tip on the bisecting line. It therefore corresponds to the load powers $(P, Q)$ on the bifurcation set. If the present load demand is indicated by point $a$ in Fig. 6, for example, which yields the voltage solutions $\boldsymbol{v}$ and $\boldsymbol{V}$ of Fig. 7a, then point $b$ is the point on the bifurcation set that corresponds to $(V+v) / 2$. The index VIPI(a) is a measure of the distance between $\mathbf{a}$ and $\mathbf{b}$. But $\mathbf{b}$ is not necessarily the point on the bifurcation set that is closest to a.

In a multi-node power network, there may be more than two voltage solutions for each given load pattern. Each voltage solution is a collection of nodal voltages.

The $L$ index can be defined for each load node by retaining the load and replacing the rest of the power network by its Thevenin equivalent, so that the simple system of Fig. $1 \mathrm{can}$ be applied. This is done for each load node $i$, and the overall index is defined as $L$ $=\max L_{i}$ (the largest of the $L_{i}$ 's), where $L_{i}$ is the index for each load node, $i$. The individual node indices tend to form clusters as the system loading changes, which can provide a good indication of which nodes will collapse together. Experiments show that $L=\max L_{i}<0.2$ is a good criterion for safe operation. When the threshold of 0.2 is exceeded, the index increases rapidly with small increases in loading. To sum up, the $L$-index method requires only the normal voltage solution, but many individual indices must be computed.

The VIPI method requires an additional voltage solution that corresponds to the point $\mathbf{b}$ on the bifurcation set. Finding such a solution for a large-scale network is difficult because it is necessary to solve for and select from many roots of a set of nonlinear algebraic equations. Fortunately, there is a method for tracking the solutions. The average of the two voltage solutions, i.e., the 
stable one and its closest counterpart, is taken just as in the case of the simple system. The loading supported by the averaged voltage is then computed and used to obtain the index. Apparently, the closer the VIPI index is to 0 , the closer the load demand is to the bifurcation set.

\section{Dynamic Mechanisms}

We will now reconstruct a voltage-collapse scenario based on the interaction among dynamic mechanisms. These mechanisms are related to the essential components of a power network: generator, voltage controller, and load. Perhaps the best way to explain their interaction is to use the simple system of Fig. 1 again. In this circuit, the voltage controller is a tap-changing transformer whose turns ratio is $1: n$. As operating conditions vary, such as a change in load, the load voltage, $V_{L}$, deviates from its nominal value and the automatic adjustment of $n$ attempts to bring the voltage back to normal.

The system behavior is completely characterized by the two quantities, $n$ and $V_{L}$, so it is sufficient to study the dynamics in the $n-V_{L}$ plane. We'll begin by studying the influence of each individual mechanism on the movement in the $n-V_{L}$ plane and then use the combined effect to explain how a

\section{The focus is on two issues, of which the first is modeling}

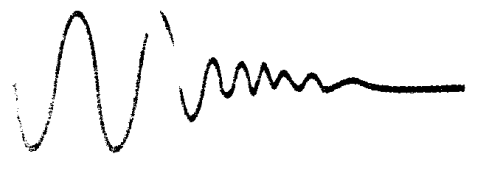

voltage collapse can arise. The $n-V_{L}$ plane is partitioned based on the influence of each mechanism (Fig. 8):

Tap-changing: The region above Curve I corresponds to $n \downarrow$ that is, reducing the transformer turns ratio when the voltage across the load is higher than the desired value $V_{o}$ - and the region below Curve I to $n \uparrow$

Load dynamics: The leaf-like region enclosed by Curve II is associated with voltage recovery. We'll call this region the leaf, with $V_{L} \uparrow$ inside the leaf and $V_{L} \downarrow$ outside. The leaf is not stationary; in fact, it is dependent on the system conditions. The leaf

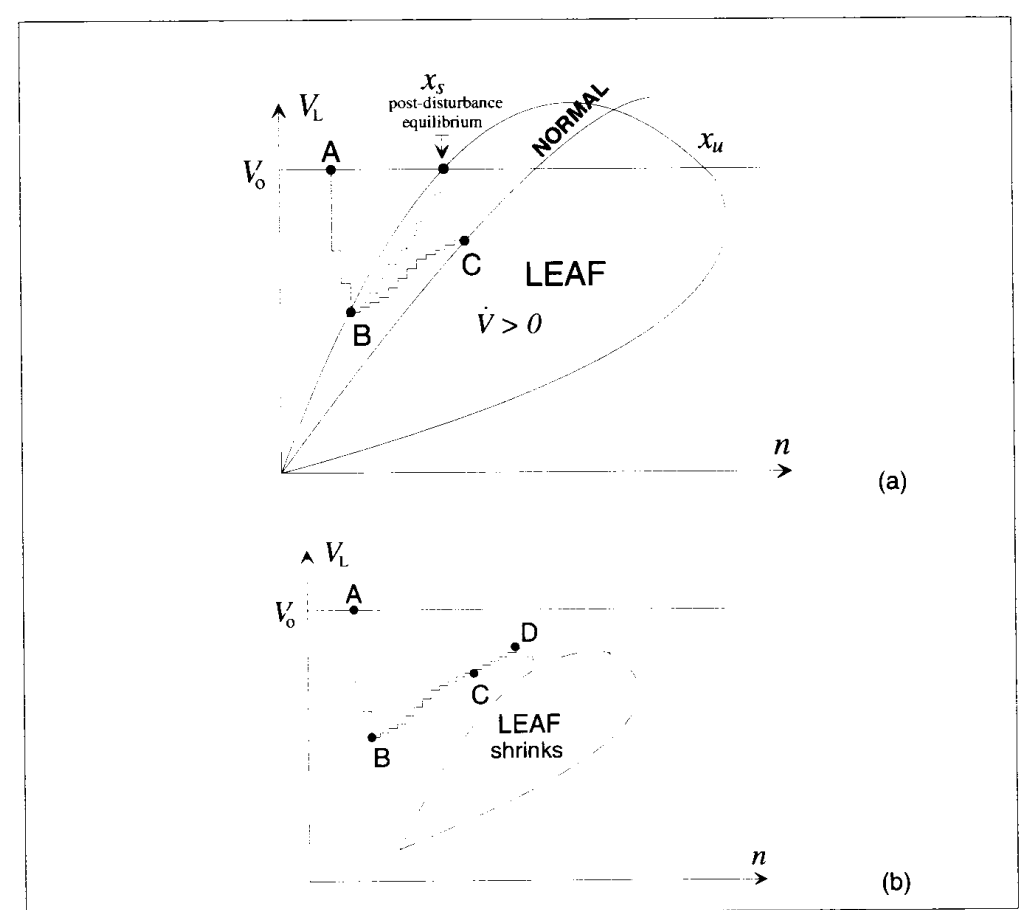

9. A scenario of voltage collapse.

is particularly dependent on the generator voltage, $E$; it shrinks as $E$ decreases. Physically, this means that the lower the generator voltage, the harder it is to maintain the load voltage at a desired level.

The intersections of Curve I and Curve II correspond to situation in which the dynamics of tap-changing and load have both subsided; that is, equilibrium conditions. These equilibria are related to the $x_{S}$ (stable) and $x_{u}$ (unstable) previously discussed.

Generator limits: Normal operation of the generator is associated with the region above the curve marked "normal." That is, when the system state is above this curve, the generator is operating at its nominal voltage without violating any constraints. But when the state crosses the boundary curve at least one of the generator's limits, stator or rotor current, is reached. As a result, the generator will be operating at a reduced voltage.

With this background, we can now provide an explanation of a voltage-collapse scenario. Assume that a system has been operating in a steady state, point $\mathbf{A}$, when a disturbance such as the sudden switching of a transmission line occurs (Fig. 9a). As a result of this disturbance, a new leaf forms. Since the operating point $\mathbf{A}$ is now outside the leaf, we have $V_{L} \downarrow$, the system moves down in the plane, and the low voltage initiates tap-changing operation - the state jumps from one vertical segment to the next. At point $\mathbf{B}$, the system state enters the leaf, and we have $V_{L} \uparrow$. If the system state does not cross the normal limit curve, it would trace a path such as the dotted one branching from point $B$. In this case, the system would settle at a new equilibrium. If, on the other hand, the system state crosses the limit curve at a point such as $\mathbf{C}$, the generator voltage $E$ decreases. The reduction in $E$ causes the leaf to shrink, and it may continue to shrink until the trajectory moves outside the leaf (Fig. 9b). Upon leaving the leaf at point $\mathbf{D}$, the system state continually heads for the direction of $V_{L} \downarrow$ : voltage collapse.

Conceptually, the interaction among mechanisms is related to bifurcation. Earlier, we considered a bifurcation resulting from slow changes in system parameters. The analysis of mechanisms in the present example permits the consideration of large disturbances such as line switching. Such disturbances cause the equilibria $x_{s}$ and $x_{u}$ to move toward each other because of the shrinkage of the leaf. This implies that the stability region also changes (Fig. 10). (Any 


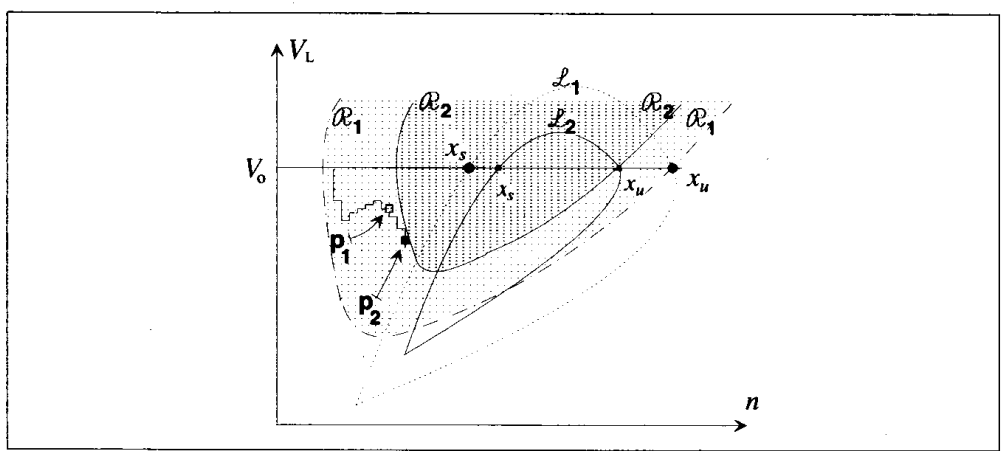

10. A shrinking stability region.

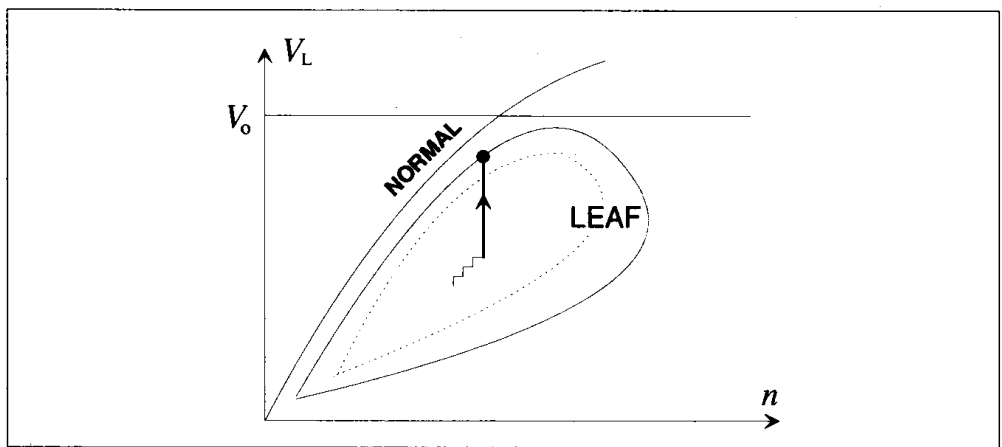

11. Locking the tap-changer while the system in the leaf allows the voltage to recover.

trajectory starting inside the stability region eventually converges to the stable equilibrium.) When the system state reaches point $\mathbf{p}_{1}$, the leaf is $\mathcal{L}_{1}$, and the stability region is $\Re_{1}$. By the time the state arrives at p2, the leaf has shrunk to $L_{2}$ and the stability region to $\Re_{2}$. Observe that $\mathbf{p}_{1}$ is inside the stability region $\Re_{1}$, but $\mathbf{p}_{2}$ is outside $\Re_{2}$. The two equilibria eventually collide (bifurcation) and vanish, along with the whole stability region.

When the system state is inside the leaf, the voltage increase indicates that the system is moving toward normal operation of the generator (Fig. 9a). However, the movement that corresponds to a monotonic fall of nodal voltages; that is, a voltage-collapse region. When tap-changers attempt to correct a lowvoltage profile, the generators are moved closer to their limit(s). If the limit is reached, the stability region begins to shrink and the voltage-collapse region expands. Voltage collapse takes place when the system state falls into an expanding collapse region. A special case of this theory is when bifurcation occurs and leads to loss of equilibrium. Then, the whole state-space becomes the collapse region.

\section{Acknowledgment}

I. Dobson is supported in part from NSF grants ECS-9009079 and ECS-9157192.C.C. Liu and $K$. Vu are supported by NSF grant ECS-8657671.K. Vu also acknowledges the support from the Clemson University Electric Power Research Association (CUEPRA). The authors would like to thank Ken Werner, $C \& D$ Technical Editor, for his helpful comments.

CD

Ian Dobson (University of Wisconsin, Madison), Hans Glavitsch (Swiss Fed. Inst. of Tech., Switzerland), Chen-Ching Liu (University of Washington), Yasuo Tamura (Waseda University, Japan), and Khoi $V u$ (Clemson University) are the members of the Subcommittee on Special Publications of the IEEE Circuits and Systems Society's Technical Committee on Power Systems \& Power Electronics \& Circuits.

\section{References}

1. Y. Mansour, ed., Voltage Stability of Power Systems: Concepts, Analytical Tools and Industry Experience, IEEE Power Systems Engineering Committee, 1991.

2. L. H. Fink, ed., Proceedings of Bulk Power Voltage Phenomena-Woltage Stability and Security, EPRI EL-6183 (January, 1989).

3. L. H. Fink, ed., Bulk Power System Voltage Phenomena II, Deep Creek Lake, Maryland (August, 1991). 